\title{
A Comparison between the Hybrid Using Genetic Algorithm and the Pure Hybrid Watermarking Scheme
}

\author{
Didi Rosiyadi, Member, IACSIT, Shi-Jinn Horng, Nana Suryana, and Nurhayati Masthurah
}

\begin{abstract}
The comparison between the hybrid watermarking using genetic algorithm and the pure hybrid DCT-SVD (Discrete Cosine Transform-Singular Value Decomposition) is presented. The watermark scheme is based on DCT-SVD]; applying DCT to the host image, map the DCT coefficient in a zigzag order, then propose the SVD on the sub-block and the singular value in each sub block that will be modified to embed the watermark image. Afterward the image quality can be improved with the GA-based evolution. Experimental results are shown the best extracted watermark quality of hybrid DCT-SVD between the hybrid using genetic algorithm and pure hybrid DCT-SVD.
\end{abstract}

Index Terms-Hybrid, genetic algorithm, DCT, SVD.

\section{INTRODUCTION}

Digital watermarking is a process of embedding data into a host data such as image, video or audio. The benefit of digital watermark are illegal distribution of digital data, copyright protection, copying of discourage unauthorized etc [1]. The important requirements of digital watermarking consist of robustness, unambiguity, perceptual transparency and capacity [2]. Robustness is a capability of watermark to various modifications or manipulations as well as cropping, filtering, noising, compression etc; unambiguity is umbigious after the extraction process; perceptual transparency is perceptual invisible after embedding process; capacity is put in some data into host image without loosing transparency. Watermarking scheme categories are permanence, visibility and domain. The watermark scheme based on domain transform has several advantages, these are perceptibility and robustness [3]-[5].

In this paper, we carried out the scheme based on DCT-SVD; propose DCT to the host image, mapping the DCT coefficient in a zigzag order; and then applying the SVD on the sub-block and the singular value in each sub block that will be modified to embed the watermark image. Afterward the image quality can be improved with the GA-based evolution. Our experiment use the peak signal to

Manuscript received March 23, 2012; revised May 6, 2012. This work was supported in part by the DIPA Research in Indonesian Institute of Sciences.

Didi Rosiyadi and Shi-Jinn Horng are with the Department of Computer Science and Information Engineering, National Taiwan University of Science and Technology (e-mail: didi.rosiyadi@gmail.com, horngsj@yahoo.com.tw).

Nana Suryana and Nurhayati Masthurah are with the Research Center for Informatics-Indonesian Institute of Sciences Jl. Cisitu, Sangkuriang, Bandung, West Java, Indonesia (e-mail: nana@suryana.or.id, masthurah@informatika.lipi.go.id, Phone. +62-22-2504711). noise ratio (PSNR) to measure the quality of the watermarked image and the correlation coefficient to verify the existence of the watermark. Experimental results are shown the best extracted watermark quality of hybrid DCT-SVD between the hybrid using genetic algorithm and pure hybrid DCT-SVD.

\section{The PROPOSED METHOD}

The proposed method of watermark scheme is introduced. The watermark scheme based on DCT-SVD; one of the advantages is durability to a variety of attacks. According to description in [6]-[10], the watermark embedding and extraction can be seen as follows.

\section{A. Watermark Embedding}

- Apply the DCT and Divide the host image into four quadrants ( SB1, SB2, SB3 and SB4)

- Scan the DCT coefficient in the zigzag manner

- Perform SVD operation for each quadrant

- Perform DCT SVD operation for watermark

- Insert the Watermark for each quadrant

- Modified the coefficient back to their original positions

- Apply the Inverse DCT to produce the watermarked host image.

\section{B. Watermark Extraction}

- Apply the DCT and Divide the host image into four quadrants

- scan the DCT coefficient in the zigzag manner

- Apply SVD operation for each quadrant

- Extract the singular values for each quadrant

- Construct the DCT coefficient for 4-visual watermarks

\section{Optimization Process by Genetic Algorithm}

- We embed the watermark in 4 different embedding positions. The coordinate of embedding position are $g$ and $h(g, h)$ in an $8 \times 8$ block. A coordinate $\mathrm{g}$ and h can represent by 6 bits and then one binary string has 24 bits, as illustrated 100111010111001010110001. For each embedding position decide the optimal value of scaling factor key.

- The Initialization is a process to create a population of chromosomes and to initiate the vectors randomly for chromosomes in each block.

- Fitness function value. In this stage the children chromosomes are generated in accordance with fitness function value. Then to measure the fitness function value is defined as follow :

$$
f_{i}=\frac{1}{n} \sum_{i=1}^{n}\left(W I\left(W^{j}, W_{i}^{* j}\right)-W E\left(I^{j}, I_{w}^{* j}\right)\right)
$$


Where $W I$ are correlation between original and watermarked image, $W E$ are correlation the original watermark and the extracted image. Then $f_{i}$ and $n$ represents fitness value and the number of attacking methods, respectively. After that, we use the roulette-wheel method to generate offspring. The highest objective function value of Parent generation produces one or more offspring.

- We use two genetic Operators. These are crossover and mutation. The Crossover stage is mate two chromosomes to produce the next generation (randomly select the crossover point after the third gene and Swap the second parts of chromosomes); Mutation is Alter one or more genes with the probability equal to the mutation rate.

\section{EXPERIMENTAL RESULT}

Generally the experiment is based on [10], we just add a sub experiment about comparison between hybrid using genetic algorithm and pure hybrid DCT-SVD. In these experiments, we use the peak signal to noise ratio (PSNR) to measure the quality of the watermarked image and the correlation coefficient to verify the existence of the watermark.

$$
\begin{gathered}
P S N R=10 \cdot \log _{10}\left(255^{2} / M S E+e p s\right) \\
\infty(V, W)=\frac{\sum_{J=1}^{k}\left(V_{(j)}-(\text { Vmean }, k) \cdot\left(W_{(j)}-(\text { Wmean }, k)\right.\right.}{\sum_{j=1}^{k} \sqrt{\left(\left(V_{(j)}-\text { Vmean }, k\right) \cdot\left(W_{(j)}-\text { Wmean }, k\right)\right)^{2}}}
\end{gathered}
$$

In which $\mathrm{V}=$ double $(\mathrm{V})$ was original singular value, $\mathrm{W}=$ double (W) is the extracted singular value, Vmean is the mean of original singular value, Wmean is the mean of extracted singular value and $\mathrm{k}$ is $(\mathrm{M}, \mathrm{N})$.

To assess the performance of the watermark scheme, several experiments were conducted using the 256x1024 gray scale host image of Indonesian Institute of Sciences (IIS) document header, the size of visual watermark IIS logo image is $256 \times 256$, watermarked of IIS document header and extracted watermark IIS logo image that are illustrated in Fig. 1.

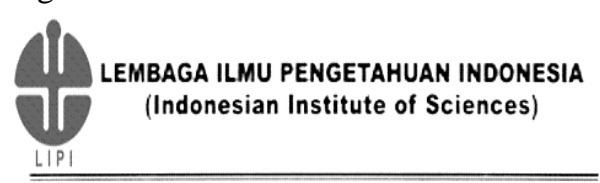

(a)

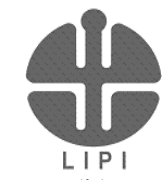

(b)

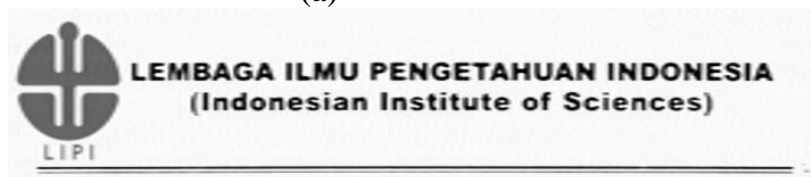

(c)

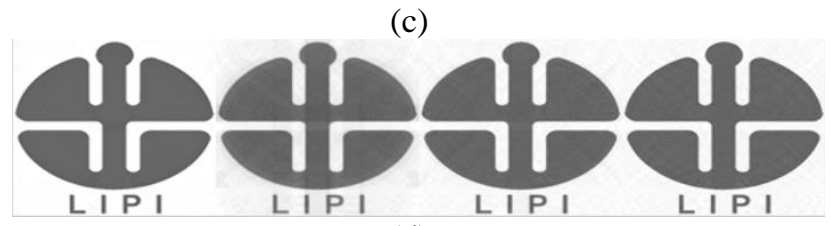

(d)
Fig. 1. (a) Host image of IIS document header, (b) Watermark of IIS logo, (c) Watermarked IIS document header (d) Extracted Watermarks

The selection of tuning parameters consists of population size is 30 , crossover rate is 0.8 , mutation rate is 0.01 , generation size is 100 . The final results of scaling factor key optimization for each sub blocks are Scaling factor key of sub blocks 1 is -0.99 , and 0.01 for the other three sub blocks. The chosen attacks were cropping on right-half with replacement, JPEG 50, rotation 60, median filtering, gaussian noise 0.5 . Table I shows the extracted watermarks for hybrid DCT-SVD using genetic algorithm after the several attacks. In this table be found seven varieties of attacks, correlation coefficient value, sub block and extracted watermark. The correlation coefficient value indicate correlation the original and extracted vector for singular values and singular values for each sub block, respectively.

TABLE I: EXTRACTED WATERMARKS OF HYBRID DCT-SVD USING

\begin{tabular}{|c|c|c|c|}
\hline Variety of Attack & $\begin{array}{l}\text { Correlation } \\
\text { Coefficient } \\
\text { value } \\
\end{array}$ & $\begin{array}{l}\text { Sub } \\
\text { block }\end{array}$ & $\begin{array}{l}\text { Extracted } \\
\text { Watermark }\end{array}$ \\
\hline $\begin{array}{lr}\text { Cropping } & \text { on } \\
\text { Right-half } & \text { with } \\
\text { Replacement } & \\
\end{array}$ & 0.9882 & 1 & \\
\hline JPEG 50 & 0.9753 & 1 & \\
\hline Rotation 60 & 0.8144 & 3 & \\
\hline Median Filtering & 0.5828 & 4 & \\
\hline $\begin{array}{l}\text { Gaussian Noise } \\
0.5\end{array}$ & 0.6024 & 4 & D皮, \\
\hline
\end{tabular}
GENETIC ALGORITHM

We now compare our experiment results in table I with those obtained from a pure hybrid DCT-SVD scheme. The pure hybrid DCT-SVD scheme were conducted using the $512 \times 512$ gray scale host image of Indonesian Institute of Sciences (IIS) document header, the size of visual watermark IIS logo image is $256 \times 256$. Table II shows the extracted watermarks for pure hybrid DCT-SVD with the same variety attacks.

TABLE II: SHOWS THE EXTRACTED WATERMARKS FOR PURE HYBRID DCT-SVD

\begin{tabular}{|l|l|l|l|}
\hline Variety of Attack & $\begin{array}{l}\text { Correlation } \\
\text { Coefficient } \\
\text { value }\end{array}$ & $\begin{array}{l}\text { Sub } \\
\text { block }\end{array}$ & $\begin{array}{l}\text { Extracted } \\
\text { Watermark }\end{array}$ \\
\hline $\begin{array}{l}\text { Cropping on } \\
\text { Right-half with } \\
\text { Replacement }\end{array}$ & -0.2067 & 1 & \\
\hline
\end{tabular}




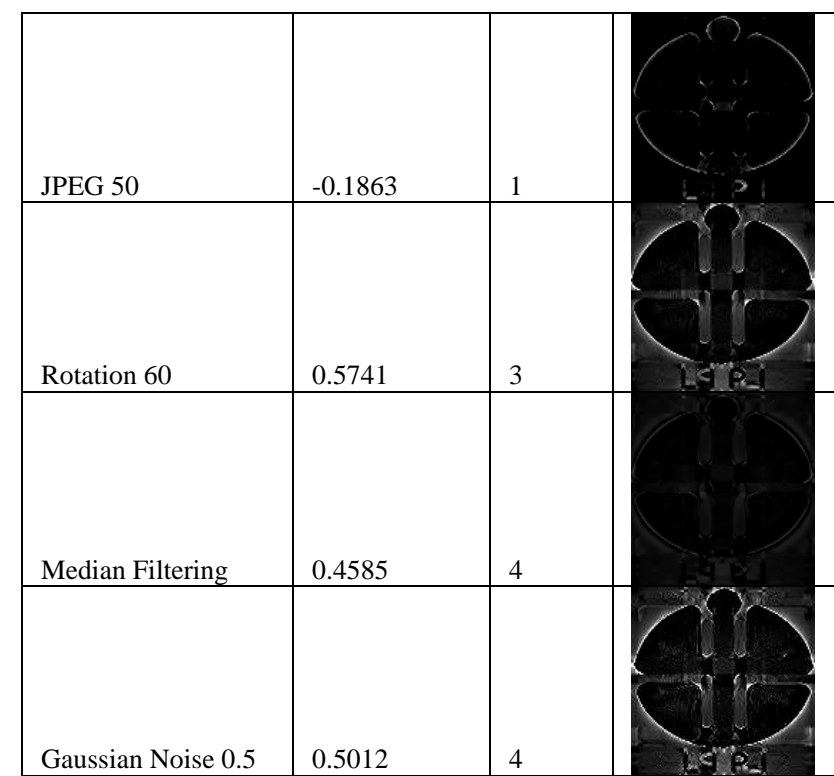

The value of all correlation coefficients and the visual quality of all images in table II are lower and worse than the result in table I, respectively. In figure 2 shows the comparison between the hybrid using genetic algorithm and the pure hybrid DCT-SVD, where the value of correlation coefficients of pure hybrid DCT-SVD is lower than the hybrid using genetic algorithm too. These conditions indicate that the extracted watermark quality of the hybrid using genetic algorithm is better than the extracted watermark quality of pure hybrid DCT-SVD.

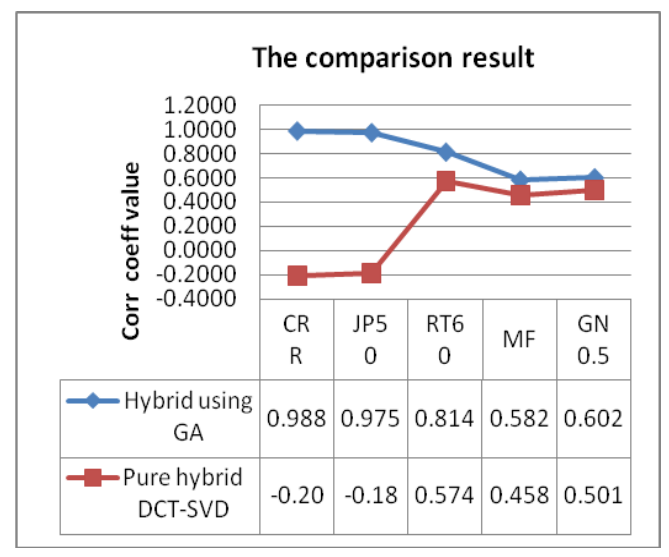

Fig. 2. The comparison result

\section{CONCLUSIONS}

In this paper, comparison between a hybrid using genetic algorithm and a pure hybrid DCT-SVD is presented. We use compare the extracted watermark quality between the hybrid using genetic algorithm and pure hybrid DCT-SVD.

Experiment show robustness of the proposed scheme to various attacks and use the peak signal to noise ratio (PSNR) to measure the quality of the watermarked image and the correlation coefficient to verify the existence of the watermark.

The result of experiment produce the hybrid DCT-SVD using genetic algorithm is better than pure hybrid DCT-SVD. The extracted watermark quality of the hybrid using genetic algorithm is better than the extracted watermark quality of pure hybrid DCT-SVD.

\section{ACKNOWLEDGMENTS}

Our thanks to Indonesian Institute of Sciences for supporting to this research and so the research paper has been written.

\section{REFERENCES}

[1] A. Sverdlov, S. Dexter, and A. M. Eskicioglu, "Robust DCT-SVD domain image watermarking for copyright protection: embedding data in all frequency," in Proc. 13th European Signal Processing Conference, Antalya, Turkey, 2005.

[2] C.-H. Huang and J.-L. Wu, "A Watermark Optimization Technique Based on Genetic Algorithms," in Proc. SPIE Visual Communications and Image Processing vol. 3971, 2000.

[3] V. Aslantas, "A SVD based digital image watermarking using genetic algorithm," International Journal of Electronics and Communications, pp. 1-4, 2007.

[4] C.-C. Lai, H.-C. Huang, and C.-C. Tsai, "ImageWatermarking Scheme Using Singular Value Decomposition and Micro-genetic Algorithm," in Proc. IEEE International Conference on Intelligent Information Hiding and Multimedia Signal Processing, 2008, pp. 469-472.

[5] F. H. Huang and Z. H. Guan, "A Hybrid SVD-DCT Watermarking Method Based on LPSNR," Pattern Recognition Letter, pp. 1769-1775, 2004.

[6] Z.-M. Lu, H.-Y. Zheng, and J.-W. Huang, "A Digital Watermarking Scheme Based on DCT and SVD," in Proc. $3^{\text {rd }}$ on Intelligent Information Hiding and Multimedia Signal Processing, Taiwan, 2007

[7] J. Holland, Adaptation in Natural and Artificial Systems, MIT press, Bradford Book Editions, Cambridge, Massachussetts, London, England, 1975.

[8] D. Goldberg, Genetic Algorithms in Search, Optimization, and Machine Learning, addison, wisely publishing company, 1989.

[9] Y.-I. Tung, Application of Computational Intelligence in Engineering course, Second semester, National Taiwan University of Science and Tech, Taiwan, 2009

[10] S.-J. Horng, D. Rosiyadi, P. Fan, X. Wang, M. K. Khan, and Y. Pan, "An efficient copyright protection scheme for e-government document images," Accepted on IEEE Multimedia, Aug. 2011.
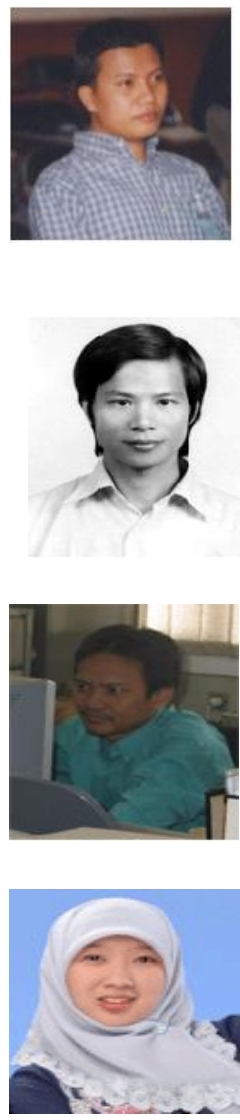

Didi Rosiyadi received the Bachelor degree in the Information System, STMIK Bandung, Indonesia and the Master degree in the Computer Science, Gadjah Mada University, Yogyakarta, Indonesia in 2001 and 2003. Currently, he is a researcher, Information System Department, Research Center for Informatics, Indonesian Institute of Sciences.

Shi-Jinn Horng received his $\mathrm{PhD}$. degree in computer science from the National Tsing Hua University in 1989. Currently, he is a professor in the Department of Computer Science and Information Engineering, National Taiwan University of Science and Technology. His research interests include multimedia, biometrics, and parallel algorithms.

Nana Suryana received the Bachelor and the Master degrees from STMIK Bandung and ITB, Indonesia, respectively. $\mathrm{He}$ is a researcher, Information System Department, Research Center for Informatics, Indonesian Institute of Sciences.

Nurhayati Masthurah received the Bachelor and the Master degrees from UGM, Indonesia, respectively. She is a researcher, Information System Department, Research Center for Informatics, Indonesian Institute of Sciences. 